\section{Nur wenige Nävi? Hautkrebsvorsorge lohnt sich trotzdem}

\begin{abstract}
Personen mit vielen Nävi haben ein erhöhtes Melanomrisiko. Allerdings werden bei ihnen fast nur dünne Tumoren entdeckt. Dagegen ist bei Melanompatienten mit wenigen Nävi das Risiko für dicke Geschwülste deutlich erhöht.
\end{abstract}

$\mathrm{P}_{\mathrm{s}}^{\mathrm{e}}$ rsonen mit multiplen sowie atypischen Nävi haben bekanntlich ein erhöhtes Melanomrisiko. Allerdings ist unklar, ob sie damit auch eine schlechtere Prognose haben. Nach einer aktuellen Analyse gehen US-Dermatologen davon aus, dass Melanome bei Personen mit mehr als 50 Nävi zum Zeitpunkt der Tumordiagnose eher noch dünn sind. Dagegen ist bei wenigen und bei atypischen Nävi das Risiko deutlich erhöht, bei der Diagnose einen Tumor jenseits von $2 \mathrm{~mm}$ Dicke zu tragen. Möglicherweise sind sich Personen mit multiplen Nävi sowie deren Ärzte des Erkrankungsrisikos eher bewusst und kontrollieren ihre Leberflecke strenger.

Für die Studie wurden 566 Melanompatienten unmittelbar nach der Diagnose untersucht. Jeweils zwei Experten ermittelten unabhängig voneinander die Gesamtzahl der Nävi sowie der atypischen Nävi. Zudem erfassten sie die Tumordicke, wobei die Patienten grob in solche mit mehr oder weniger als $2 \mathrm{~mm}$ dicken Tumoren eingeteilt wurden. Da in der Regel die meisten Patienten dün- nere Tumoren hatten, wurde ein überproportional großer Anteil mit dicken Tumoren in die Studie aufgenommen.

Im Schnitt lag das Alter der Patienten bei 57 Jahren, rund $40 \%$ waren weiblich. Wenig überraschend hatten die jüngsten Patienten die meisten Nävi - das spiegelt das hohe Erkrankungsrisiko wieder. Mehr als 50 Nävi wurden bei $28 \%$ der Patienten unter 45 Jahren beobachtet, aber nur bei knapp $3 \%$ derjenigen im Alter von 70 oder mehr Jahren. Letztere hatten zu $88 \%$ weniger als 20 Nävi. Ein ähnliches Bild ergab sich bei den Patienten mit atypischen Flecken: Mehr als fünf atypische Nävi hatten $27 \%$ der jüngsten, aber nur $1,4 \%$ in der ältesten Patientengruppe. Von Letzteren zeigten $93 \%$ überhaupt keine atypischen Nävi, von den Jüngsten nur die Hälfte. Generell stieg die Zahl der atypischen Nävi mit der Gesamtzahl der Leberflecke.

Wurde die Tumordicke analysiert, so hatten die Patienten mit den meisten Nävi die besten Aussichten: Bei ihnen waren die Tumoren im Schnitt nur 1,1 mm dick, bei jenen mit weniger als 20 Flecken lag die Dicke hingegen bei 2,3 mm. Dieser Zusammenhang ist allerdings stark altersabhängig und gilt vor allem für $\mathrm{Pa}$ tienten unter 60 Jahren. Haben sie mehr als 50 Nävi, dann ist die Wahrscheinlichkeit, einen dicken $(>2 \mathrm{~mm})$ statt eines dünnen Tumors zu haben, um $65 \%$ geringer als bei solchen mit weniger als 20 Nävi (Odds Ratio $=0,35)-$ und zwar auch dann, wenn eine Reihe von Faktoren wie Geschlecht, anatomische Position oder histologischer Subtyp berücksichtigt werden. Umgekehrt ist die Chance für einen dicken Tumor um das 2,4-Fache erhöht, wenn bei den jüngeren Patienten mehr als fünf atypische Nävi beobachtet werden. Bei Patienten über 60 Jahren zeigt sich jedoch ein anderer Zusammenhang: Hier ist bei mehr als fünf atypischen Nävi das Risiko für einen dicken versus dünnen Tumor um rund $80 \%$ reduziert (Odds Ratio $=0,22$ ).

Fazit: Die allermeisten Melanompatienten haben weniger als 20 Nävi. Gerade bei ihnen finden Ärzte aber vermehrt dicke Tumoren. Dermatologen sollten daher Personen mit wenigen Nävi genau so kritisch betrachten und untersuchen wie solche mit sehr vielen - vor allem jenseits des 60. Lebensjahrs, so die Autoren der Studie.

Thomas Müller

Geller A C et al. Total Nevi, Atypical Nevi, and Melanoma Thickness. An Analysis of 566

Patients at 2 US Centers. JAMA Dermatol 2016; 152: 413-8

\title{
Ist das überhaupt noch derselbe Mann?
}

Wegen allgemeiner Schwäche wurde ein 76-jähriger Mann ins Krankenhaus aufgenommen. Einige Wochen zuvor war ihm eine zunehmende graublaue Verfärbung der gesamten Haut aufgefallen. Diese war so ausgeprägt, dass er nicht mehr anhand seines Personalausweises zu erkennen war. 14 Monate zuvor war ein malignes Melanom diagnostiziert worden, das BRAF-negativ war.

Fünf Monate vor der Vorstellung wurden multiple Metastasen in Leber und Milz festgestellt. Der Mann wurde mit vier Zyklen Dacarbazin behandelt. Die ausgeprägte Hyperpigmentierung der Haut war Ausdruck einer diffusen Melanosis cutis. Diese Veränderung ist ein prognostisch ungünstiges Zeichen und ist mit einer durchschnittlichen Überlebenszeit von etwa sechs Monaten assoziiert. Der Patient lebte aber nach Auftreten der Melanosis cutis noch 16 Monate, möglicherweise als Ergebnis einer Immuntherapie mit Ipilimumab. Prof. Hermann S. FüeßI

Jansen T \& Hoff NP. Diffuse melanosis cutis. N Engl J Med 2016; 374: 1177

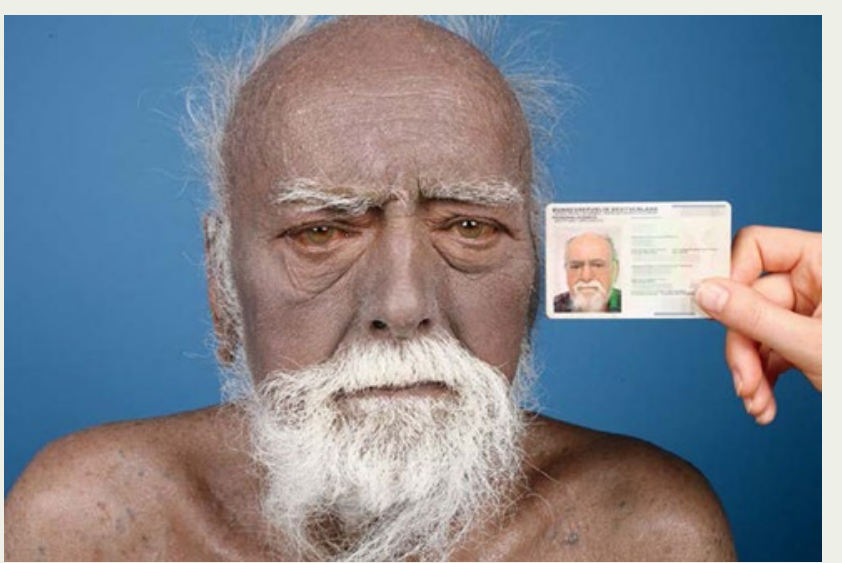

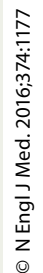

Ausgeprägte Hyperpigmentierung der gesamten Haut; Personalausweis zum Vergleich. 